\title{
Sample Timing, diagnosis of Subclinical Thyroid Dysfunction and Mortality in Acute
}

\section{Myocardial Infarction: ThyrAMI1 study}

Salman Razvi, MD, $\mathrm{FRCP}^{1,2}$; Owain Leng, $\mathrm{MD}^{3}$; Avais Jabbar, $\mathrm{MD}^{1,3}$; Arjola Bano, MD, $\mathrm{PhD}^{4}$; Lorna Ingoe, $\mathrm{BSC}^{3}$; Caroline Addison, FRCPath ${ }^{2}$; Honey Thomas, $\mathrm{MD}^{5}$; Peter Carey, $\mathrm{MD}^{6}$; Shahid Junejo, $\mathrm{MD}^{6}$; David Austin, MD ${ }^{7}$; John P. Greenwood, MD, PhD ${ }^{8}$; Azfar Zaman, $\mathrm{MD}^{3,9}$.

\section{${ }^{1}$ Institute of Genetic Medicine, Newcastle University, Newcastle upon Tyne, UK}

${ }^{2}$ Gateshead Health NHS Foundation Trust, Gateshead, UK

${ }^{3}$ Newcastle upon Tyne Hospitals NHS Foundation Trust, Newcastle upon Tyne, UK

${ }^{4}$ Institute of Social and Preventive Medicine, University of Bern, Bern, Switzerland

${ }^{5}$ Department of Cardiology, Northumbria Healthcare NHS Foundation Trust, Ashington, UK

${ }^{6}$ City Hospitals Sunderland NHS Foundation Trust, Sunderland, UK

${ }^{7}$ The James Cook University Hospital, Middlesbrough, UK

${ }^{8}$ Leeds Institute of Cardiovascular and Metabolic Medicine, University of Leeds, UK

${ }^{9}$ Institute of Cellular Medicine, Newcastle University, Newcastle upon Tyne, UK

\section{Correspondence to:}

Dr S Razvi

Senior Lecturer and Consultant Endocrinologist

Institute of Genetic Medicine, Newcastle University

Newcastle upon Tyne NE1 3BZ, United Kingdom

Ph: +44 1912418838; Email: salman.razvi@ncl.ac.uk

(C) Endocrine Society 2019. All rights reserved. For permissions, please e-mail: journals.permissions@oup.com. jc.2019-01673. See endocrine.org/publications for Accepted Manuscript disclaimer and additional information. 


\section{Disclosure statement}

Dr Razvi has received speaker fees from Merck plc and Abbott Pharmaceuticals India Ltd, makers of levothyroxine. None of the other authors have any conflicts of interest or anything relevant to disclose.

\section{Funding}

SR is funded by a National Institute for Health Research Career Development Fellowship (CDF-2012-05-231). The views expressed in this publication are those of the author(s) and not necessarily those of the NHS, the National Institute for Health Research or the Department of Health and Social Care. The funder was not engaged in study design; in the collection, analysis and interpretation of data; in the writing of the report; and in the decision to submit the article for publication. 


\section{Abstract}

Objective: The objective of this study was to determine the impact of blood sample timing on the diagnosis of subclinical thyroid dysfunction (SCTD) and mortality in patients with acute myocardial infarction (AMI).

Patients, Design and Main Outcome Measures: Patients with AMI had thyroid function evaluated on admission between December 2014 and December 2016 and those with abnormal serum TSH had repeat thyroid function assessed at least a week later. The association between sample timing and SCTD was evaluated by logistic regression analysis. Secondary outcomes were confirmation of SCTD on repeat testing and all-cause mortality up to June 2018.

Results: Of the 1806 patients [29.2\% women, mean ( $\pm S D)$ age of $64.2( \pm 12.1)$ years] analysed, the prevalence of subclinical hypothyroidism $(\mathrm{SCH})$ was $17.2 \%(\mathrm{n}=311)$ and subclinical hyperthyroidism (SHyper) was $1.2 \%(n=22)$ using a uniform TSH reference interval. The risk of being diagnosed with SCTD varied by sample timing in fully-adjusted models. The risk of SCH was highest between 00:01-06:00hrs and lowest between 12:0118:00hrs, $p$ for trend $<0.001$, and risk of SHyper was highest between 12:01-18:00hrs and lowest between 00:01-06:00hrs. Furthermore, time of the initial sample was associated with the risk of remaining in a $\mathrm{SCH}$ state subsequently. Mortality in $\mathrm{SCH}$ patients was not elevated when a uniform TSH reference interval was utilised. However, when time-periodspecific TSH reference ranges were utilised, the mortality risk was significantly higher in $\mathrm{SCH}$ patients with $\mathrm{HR}(95 \% \mathrm{Cl})$ of $2.26(1.01-5.19), \mathrm{p}=0.04$. 
Conclusions: Sample timing impacts on the diagnosis and prognosis of $\mathrm{SCH}$ in AMI patients. If sample timing is not accounted for, $\mathrm{SCH}$ is systemically misclassified, and its measurable influence on mortality is lost.

Keywords: Thyroid function, sample timing, acute myocardial infarction, mortality

\section{Précis}

We analyzed thyroid function sample timing in patients with acute myocardial infarction and found that sample timing effects the diagnosis of subclinical thyroid dysfunction and subsequent mortality 


\section{Introduction}

The diagnosis of subclinical thyroid dysfunction (SCTD) is established on the basis of abnormal serum thyrotropin (TSH) levels and when circulating thyroid hormone (thyroxine and triiodothyronine) concentrations are within the reference range. The log-linear relationship between TSH and thyroid hormones means that even minor alterations in thyroid hormones leads to an amplified TSH response. ${ }^{1}$ Therefore, a persistent TSH value outside its reference range indicates that thyroid hormones are abnormal for the given individual even if the levels of the thyroid hormones themselves lie within the normal range. Thus, an abnormal serum TSH measurement is essential in establishing a diagnosis of primary thyroid dysfunction and, furthermore, it implies that SCTD may be defined purely in biochemical terms. Subclinical hypothyroidism $(\mathrm{SCH})$ and subclinical hyperthyroidism (SHyper) have a prevalence ranging between $5-18 \%$ and $0.1-2 \%$, respectively, ${ }^{2}$ and both are associated with cardiovascular diseases as well as mortality in some but not all studies. ${ }^{3,4}$ Furthermore, SCTD in patients admitted with acute coronary syndromes has been linked to adverse outcomes. ${ }^{5,6}$

Serum TSH is affected by a number of factors including age, gender, ethnicity, smoking status, body mass index, iodine status, thyroid peroxidase antibody (TPOAb) status, the type of assay used, drugs and concurrent illnesses. ${ }^{7}$ Sample timing also has an important effect on serum TSH levels with a recognised circadian rhythm in serum TSH in healthy individuals reaching peak levels in the early hours of the morning and nadir in the afternoon (8). Most studies that have investigated the effect of sample timing on serum TSH and thyroid hormones have been in small groups of healthy volunteers and have shown a mild but significant fluctuation within the reference range. ${ }^{8}$ The circadian pattern of serum TSH 
secretion has been found to be diminished or absent in a variety of circumstances, including patients with critical illness, Alzheimer's disease, other endocrinopathies, and during periods of fasting. ${ }^{9}$ It has been generally accepted that the circadian fluctuation of serum TSH and thyroid hormones contributes to the width of their respective reference ranges but does not substantially affect the diagnosis of SCTD. ${ }^{10}$ Nevertheless, a recent retrospective analysis from a large laboratory database suggests that the upper limit of serum TSH levels is influenced by the sample timing time as well as age. ${ }^{11}$ It is unknown whether sample timing of thyroid function in patients presenting with acute myocardial infarction (AMI) impacts on the diagnosis of SCTD and subsequent adverse outcomes. We analysed data from the multicentre ThyrAMI-1 study to investigate if sample timing impacts on the diagnosis of SCTD in AMI patients and subsequent mortality.

\section{Methods}

Participants: ThyrAMI-1 is a prospective study that recruited patients $(n=1982)$ with both acute ST-elevation (STEMI) and non-ST-elevation (NSTEMI) myocardial infarction from six cardiac centres in England between December 2014 and December 2016. ${ }^{12}$ (Supplementary Figure 1). ${ }^{13}$ AMI was diagnosed using the standard third universal definition criteria. ${ }^{14}$ Participants provided written informed consent and the research was approved by the UK National Research Ethics Service (reference number 14/NE/0151). The aim of the project was to investigate thyroid function in patients admitted with AMI from the first available blood sample soon after admission to hospital and to identify individuals with $\mathrm{SCH}$ to recruit to an interventional trial of treatment with levothyroxine (LT4) - the ThyrAMI-2 trial. In this paper we report the results of the observational ThyrAMI-1 study only. On admission to 
hospital blood samples were analysed for routine clinical parameters and thyroid function was evaluated after the participant had provided consent. All samples were obtained prior to invasive coronary procedures. Participants who had samples for thyroid function obtained more than 24 hours after presentation (due to insufficient sample volume in the first available sample) were excluded from this analysis $(n=48)$. In addition, patients with newly-diagnosed overt thyroid disease $(n=6)$ and those on medications affecting thyroid function such as amiodarone, lithium and oral glucocorticoids $(n=9)$ were excluded. Participants treated with LT4 $(n=113)$, however, were included and a sensitivity analysis was conducted after their exclusion. Of the remainder, those with abnormal TSH values and normal thyroid hormone levels were classed as having SCTD. The time of blood sampling was acquired from the electronic laboratory database at each site and was defined as the time when the blood sample was collected (reported in hh:mm format). This information was not available for 39 participants and, for whom, time of sample receipt in each participating laboratory was utilised. The median time difference between sample collection and reception in the laboratory was 32 minutes. Patients that had evidence of SCTD on the initial sample $(n=336)$ were invited to have their thyroid function repeated $7-10$ days later between 08:00 and 10:00 hours to assess if the SCTD was persistent. Out of these, 275 individuals agreed while another 33 participants had repeat testing via their General Practitioner (Family Physician). All repeat thyroid function tests utilised the same assay as the initial investigation.

Mortality status was assessed for all participants up to $30^{\text {th }}$ June 2018 by interrogating the National Health Service (NHS) Summary Care Records (SCR) that provides up to date lifestatus for all registered patients via a unique NHS number. ${ }^{15}$ The start of the follow-up 
period was the date of the AMI and the end of follow-up was date of death or the date of ascertainment via the SCR.

Laboratory measurements: Serum TSH, FT4 and FT3 as well as creatinine were analysed using either Roche eCobas ( 4 centres) or Advia Centaur ( 2 centres) immunoassays. The reference ranges for classifying normal thyroid parameters were uniformly set a priori across all sites irrespective of immunoassay method being used. The performance of both the Roche and Centaur assays for estimating TSH show very good agreement with a previous analysis demonstrating a regression co-efficient ( $95 \%$ confidence intervals) of 0.99 (0.99-0.99). ${ }^{16}$ The reference ranges were: TSH 0.4-4.0 mU/L, FT4 10-25 pmol/L (0.78-1.94 $\mathrm{ng} / \mathrm{dL})$, FT3 3.0-7.0 pmol/L (2.28-4.23 pg/mL). Anti-thyroid peroxidase antibody (TPOAb) was measured by Roche or Centaur immunoassays and levels below $35 \mathrm{mU} / \mathrm{L}$ were classed as negative. $\mathrm{SCH}$ was defined as raised serum TSH (>4.0 mU/L) with FT4 within the reference range and SHyper was defined as low serum TSH $(<0.4 \mathrm{mU} / \mathrm{L})$ with both FT4 and FT3 within their respective reference ranges.

Statistical analyses: Continuous variables across time-periods were compared using ANOVA or Kruskal-Wallis (depending on normal or non-normal distribution, respectively) and categorical variables were compared by Fisher's exact test. The relationship and the linearity between sample timing (as a continuous parameter from 1 to 1440 minutes representing the number of minutes in 24 hours) and thyroid function parameters were examined using ordinary least squares regression with restricted cubic splines with three knots. Covariates for the multivariable models were selected based on clinical relevance or if known to affect serum TSH levels. Binary logistic regression analyses were performed separately for SCH and SHyper (and compared to euthyroidism) to evaluate the impact of 
time of sampling (in periods of 6 hours each). The relationship between SCTD and all-cause mortality was evaluated using Cox proportional hazards analysis. All analyses were adjusted for relevant variables such as age, gender, centre, smoking status (current, ex- or nonsmoker), body mass index (BMI), serum creatinine, type of AMI (STEMI or NSTEMI), pain to sampling time (calculated from onset of pain or symptoms of AMI till time of blood sampling), anti-TPOAb levels (as a continuous variable), history of ischemic heart disease (yes/no), hypertension (yes/no), diabetes mellitus (yes/no), cerebrovascular disease (yes/no), atrial fibrillation (yes/no) and hypothyroidism (yes/no). Assay type was not used as an additional covariate in these analyses as centre from where the participant was recruited (and their thyroid function measured) is already included. Lower and upper limits of timeperiod-specific reference ranges for thyroid function parameters were calculated using the $2.5^{\text {th }}$ and the $97.5^{\text {th }}$ percentile values, respectively, in patients not on LT4 treatment. ${ }^{17}$ As higher serum TSH levels per se rather than time-period specific TSH abnormalities could be linked with mortality, we also examined the relationship between baseline serum TSH and mortality using Cox proportional hazards analysis after adjusting for the abovementioned variables.

To ensure the validity of the results obtained, several sensitivity analyses were performed: analysing the relationship between sample timing and thyroid function parameters stratified by the thyroid function assays utilised, by LT4 use, and by type of AMI. The statistical software programmes SPSS v24.0 (Chic, III) and R (rms package, R project, Institute for Statistics and Mathematics, R Core Team, version 3.2.2) were utilised for regression. A p value $<0.05$ was deemed as being statistically significant for all analyses. 


\section{Results}

Prevalence of SCTD and baseline characteristics by time of sampling

The baseline characteristics of participants for each time period are presented in Table 1. The median (IQR) time interval between onset of chest pain and blood sampling was 137 (65-218) minutes. Clinical, biochemical and pre-existing medical conditions were similar across the various periods. However, there were less STEMI admissions in the 12:01-18:00 hrs period than the other periods. In addition, serum TSH and FT3 were highest between 00:01-06:00 hrs and lowest in the 12:01-18:00 period whereas there was no significant difference between FT4 levels. Consequently, the diagnosis of $\mathrm{SCH}$ was highest in the 00:0106:00 period (20.9\%) and lowest in the $12: 01-18: 00$ period ( $8.7 \%)$. Conversely, the prevalence of SHyper was lowest in the 00:01-06:00 period $(0.7 \%)$ and highest in the period between 12:01-18:00 hrs (2.5\%). When time-period specific TSH ranges were utilised (Supplementary Table 1$)^{13}$, the prevalence of $\mathrm{SCH}$ reduced dramatically $(n=49 ; 2.7 \%)$ whereas the frequency of SHyper participants increased slightly $(n=37 ; 2 \%)$. Compared to the significant variation in frequency of both $\mathrm{SCH}$ and SHyper across various periods (Table 1) the prevalence of both $\mathrm{SCH}$ and SHyper was similar across all periods when time-period specific TSH reference intervals were utilised $(p=0.79)$.

\section{Sample timing and thyroid function parameters}

Time of sampling as a continuous variable over 24 hours (from 0 to 1440 minutes) was significantly associated with serum TSH and FT3 levels in an independent and nonlinear manner (Figure 1). Serum TSH and FT3 levels were highest between 00:01-06:00 hrs and lowest between 12:01-18:00 hrs. No significant relationship was observed between time of sampling and FT4 or TPOAb levels. Furthermore, there was no statistical interaction 
between time of sampling and pain to sampling time on the various thyroid function parameters (all $p$ values $>0.10$ ).

\section{Sample timing and thyroid dysfunction}

There was a significant and non-linear association between time of sampling and risk of being diagnosed with $\mathrm{SCH}$ ( $p$ for non-linearity<0.0001). Restricted cubic spline analysis with 3 knots confirmed that the risk of being diagnosed with SCTD varied with time of sampling. The-risk of being diagnosed with $\mathrm{SCH}$ was higher in the 00:01-06:00 period and lower in the 12:01=18:00 period (Figure $2 \mathrm{~A}$ ). The other independent predictors of $\mathrm{SCH}$ were increasing age, female gender, STEMI and positive TPOAb status.

The-risk of being diagnosed with SHyper was higher in the 12:01-18:00 period and lower in the 00:01-06:00 period (Figure 2B). In addition, a significant negative association was found between SHyper and BMI.

\section{Sample timing and risk of remaining in SCTD state}

A total of 308 patients with SCTD diagnosed on admission had repeat thyroid function assessed ( $n=290$ and $n=18$ with baseline SCH and SHyper, respectively). Of these, 127 (44\%) of individuals with SCH and 11 (61\%) of participants with SHyper normalised serum TSH levels. There was a significant difference in normalisation rates depending on baseline timeperiod of sampling. In the baseline SCH group, 58\% of patients from 00:01-06:00 hrs normalised serum TSH on repeat testing whereas only 28\% of those from 12:01-18:00 normalised $(p<0.001)$ (Figure 3). In the SHyper group at baseline, no statistical analysis was performed due to the small number of participants in each time-period. The one participant with SHyper at baseline time-period of 00:01-06:00 hrs remained in SHyper state whereas 5 
participants, of the 11 with repeat data available for the 12:01-18:00 hrs period normalised their TSH.

In patients with $\mathrm{SCH}$, baseline time of sampling was associated with the risk of remaining in the $\mathrm{SCH}$ state on repeat thyroid function testing. Individuals who had initial thyroid function test drawn between 12:01-18:00 hrs were more likely to remain in the SCH state than those who had initial sampling between 00:01-06:00 hrs [OR $2.56(1.09-5.95)$ ] or between 18:01-00:00 hrs [OR $2.33(1.01-5.26)]$. Other variables that were significantly associated with remaining SCH were higher initial serum TSH level [OR $1.32(1.13-1.55)$ and TPOAb positivity [OR $2.56(1.67-3.58)]$.

All-cause mortality in relation to SCTD status determined using uniform or time-period specific TSH reference intervals.

There were 168 mortality events over a median (IQR) follow-up period of 28 (23-34) months: 31 (9.9\%) events in patients with $\mathrm{SCH}$ on admission and $135(9.2 \%)$ events in euthyroid patients. When uniform TSH reference intervals were utilized, $\mathrm{SCH}$ was not associated with all-cause mortality $(\mathrm{HR}, 1.06 ; 95 \% \mathrm{Cl}, 0.69-1.62 ; \mathrm{p}$-value, 0.80$)$.

Furthermore, all-cause mortality in the $\mathrm{SCH}$ participants was not different across the various time periods ( $\mathrm{p}$ for interaction between $\mathrm{SCH}$ and time-period $=0.34$ ). There were 2 mortality events in the SHyper group over the follow-up period.

However, mortality was significantly higher in $\mathrm{SCH}$ patients that were categorised using a time-period specific reference interval with HR of $2.26(1.01-5.19), p=0.04$. Mortality in the SHyper group was similar to euthyroid controls when time-period specific TSH reference intervals were used with HR of $0.62(0.19-1.97), p=0.42$ (Figure 4). Mortality was similar in 
SCTD patients who had persistent SCTD on repeat function (data not shown). There was no significant relationship between baseline serum TSH levels and mortality $(p=0.76)$ (Supplementary Figure 2). ${ }^{13}$

Sensitivity analysis: Several sensitivity analyses were performed to assess the validity of our results. First, we evaluated the relationship between thyroid function tests (TSH, FT4 and FT3) with time of sampling separately for the two immunoassays used (Roche and Centaur) and the results remained similar (Supplementary Table 2$).{ }^{13}$ Second, we assessed the relationship between thyroid function tests and time of sampling for the LT4 users and nonusers and found that the circadian rhythm of TSH and FT3 was not observed in LT4 users (Supplementary Table 3). Third, we investigated the association between thyroid function tests and time of sampling separately for STEMI and NSTEMI patients and found no difference (Supplementary Table 4). ${ }^{13}$ Fourth, we analysed the relationship between thyroid function tests and time of sampling after excluding those patients with type 2 diabetes who used metformin and this confirmed the circadian rhythm of serum TSH and FT3 levels although serum TSH levels tended to be slightly higher (data not shown).

\section{Discussion}

The results of this study have shown that the diagnosis of SCTD and subsequent rates of normalisation of serum TSH levels in patients with AMI is influenced by the time of sampling. Importantly, our data suggest that TSH levels higher than the time-period specific reference range are associated with increased mortality, and, crucially, this association was absent when standard TSH reference ranges are applied. These results contest the previously held view that the time of sampling has little impact on the width of the TSH 
reference range and suggest that the time of sampling should be considered when evaluating whether a given TSH result is abnormal.

Diurnal variation of serum TSH and, to a lesser extent, FT3 is recognised and is believed to contribute to the upper and lower limits of the reference range but is not considered to impact on the diagnosis of SCTD. ${ }^{18,19}$ This view is based on small studies of young healthy volunteers $^{8}$ or patients with hypothyroidism ${ }^{20}$ and may not be applicable to the general population or, those with concurrent illness. The rise in nocturnal TSH levels is considered to be due to release of more immature form of TSH that is less bioactive. ${ }^{21}$ More recently, several retrospective analyses of tens of thousands of patients confirmed that the TSH concentrations or its reference interval varies significantly depending on the time of sampling. ${ }^{11,22,23}$

An analysis of 47 patients with AMI demonstrated that mean TSH levels over 5 days were lower in non-survivors, possibly related to greater suppression of TSH by non-thyroidal illness. ${ }^{5}$ In another study involving cardiac patients, mild alterations in thyroid function, assessed within 2-5 days of hospital admission, were associated with worse prognosis, although patients with acute coronary syndromes were excluded. ${ }^{6}$ Another analysis of patients with acute coronary syndrome revealed that patients with high TSH levels $(>4 \mathrm{mU} / \mathrm{L})$ had poorer outcomes. ${ }^{24}$ It is difficult to evaluate the true association of thyroid function with outcomes in these studies as TSH levels are affected by the illness as well as its treatment. 
Our analysis has several strengths. It is a prospective analysis of a large number of participants with AMI whose data were collected in a structured manner. Samples were obtained at the first opportunity after presentation to hospital (so the impact of nonthyroidal illness was minimised) and prior to diagnostic coronary angiography (radiographic contrast contain iodine in supra-physiological amounts that could affect thyroid function). ${ }^{25}$ Moreover, we also collected data on subsequent thyroid function in patients with initial SCTD in whom the repeat samples were collected at a uniform time-period. In addition, the analyses were adjusted for other confounding variables that could impact on TSH and thyroid hormone levels. Finally, there are no published data on mortality outcomes in relation to baseline thyroid function and time of sampling. Our analysis has some limitations. First, some of the thyroid function parameters were evaluated by a different assay. However, the two TSH immunoassays utilised are known to yield similar results ${ }^{16}$ and results were similar when analyses were performed separately for the two assays. Second, additional unaccounted variables (iodine intake, fasting and stress) or unmeasured parameters may have influenced our findings. Nevertheless, a wide variety of demographic, physical and biochemical factors were included in our multivariate regression analyses. Third, we did not have data available on recurrent cardiovascular events and nor does our analysis evaluate mechanisms as to how high serum TSH may adversely impact on mortality. Fourth, the number of individuals with SHyper (diagnosed using either standard TSH reference intervals or time-period specific reference ranges) are relatively low and the results obtained for this group should be interpreted with caution. Finally, our results were obtained from patients with $\mathrm{AMI}$ and cannot be generalizable to other acute conditions or to community-dwelling individuals in whom sampling for thyroid function is usually restricted to office hours (approximately between 08:00 till 18:00 hrs). 
In conclusion, our results suggest that both the initial diagnosis of SCTD in patients with AMI and the proportion that subsequently normalise thyroid function is independently and significantly associated with time of sampling. Furthermore, mortality risk is similar in both $\mathrm{SCH}$ and euthyroid patients with $\mathrm{AMI}$ when uniform TSH reference ranges are utilised, as per current clinical practice, but, risk of mortality more than doubles when time-period specific TSH reference ranges are used to identify SCH. Our findings are important for interpretation of TSH levels in clinical practice. They suggest that for an accurate diagnosis of SCTD, the TSH reference range should take the time of sampling into account. Importantly, using this methodology to interpret the results may provide important prognostic information in patients after AMI.

\section{Acknowledgments}

The research was part-supported by the National Institute of Health and Social Care Research (NIHR) infrastructure at Leeds. 


\section{References}

1. Reichlin S, Utiger RD. Regulation of the pituitary-thyroid axis in man: relationship of TSH concentration to concentration of free and total thyroxine in plasma. J Clin Endocrinol Metab. 1967;27(2):251-255.

2. Biondi B, Cooper DS. The clinical significance of subclinical thyroid dysfunction. Endocr Rev. 2008;29(1):76-131.

3. Razvi S, Jabbar A, Pingitore A, Danzi S, Biondi B, Klein I, Peeters R, Zaman A, lervasi G. Thyroid hormones and cardiovascular function and diseases. J Am Coll Cardiol. 2018;71(16):1781-1796.

4. Jabbar A, Pingitore A, Pearce SH, Zaman A, lervasi G, Razvi S. Thyroid hormones and cardiovascular disease. Nat Rev Cardiol. 2017;14(1):39-55.

5. Friberg L, Werner S, Eggertsen G, Ahnve S. Rapid down-regulation of thyroid hormones in acute myocardial infarction: is it cardioprotective in patients with angina? Arch Intern Med. 2002;162(12):1388-1394.

6. Iervasi G, Molinaro S, Landi P, Taddei MC, Galli E, Mariana F, L'Abbate A, Pingitore A. Association between increased mortality and mild thyroid dysfunction in cardiac patients. Arch Intern Med. 2007;167(14):1526-1532.

7. Jonklaas J, Razvi S. Reference interval in the diagnosis of thyroid dysfunction: treating patients not numbers. Lancet Diabetes Endocrinol 2019. doi: $10.1016 / \mathrm{S} 2213-8587(18) 30371-1$.

8. Andersen S, Bruun NH, Pedersen KM, Laurberg P. Biologic variation is important for interpretation of thyroid function tests. Thyroid. 2003;13(11):1069-1078.

9. Roelfsema F, Veldhuis JD. Thyrotropin secretion patterns in health and disease. Endocr Rev. 2013;34(5):619-657. 
10. Lucke C, Hehrmann R, von Mayersbach K, von zur Muhlen A. Studies on circadian variations of plasma TSH, thyroxine and triiodothyronine in man. Acta Endocrinol. $1977 ; 86(1): 81-88$.

11. Ehrenkranz J, Bach PR, Snow GL, Schneider A, Lee JL, Ilstrup S, Bennett ST, Benvenga S. Circadian and circannual rhythms in thyroid hormones: determining the TSH and free T4 reference intervals based upon time of day, age, and sex. Thyroid. $2015 ; 25(8): 954-961$.

12. Jabbar A, Ingoe L, Pearce S, Zaman A, Razvi S. Thyroxine in acute myocardial infarction (ThyrAMI) - levothyroxine in subclinical hypothyroidism post-acute myocardial infarction: study protocol for a randomised controlled trial. Trials. 2015;16:115. doi: 10.1186/s13063-015-0621-5.

13. Razvi S, Leng O, Jabbar A, Bano A, Ingoe L, Addison C, Thomas H, Carey P, Junejo S, Austin D, Greenwood JP, Zaman A. From: Sample timing, diagnosis of subclinical thyroid dysfunction and mortality in Acute Myocardial Infarction:ThyrAMI1 study. Supplemental data. Deposited 11 September 2019. doi: 10.25405/data.ncl.9804116 https://data.ncl.ac.uk/articles/JCEM Supplementary data pdf/9804116

14. Thygesen K, Alpert JS, Jaffe AS, Simoons ML, Chaitman BR, White HD; Joint ESC/ACCF/AHA/WHF Task Force for the Universal Definition of Myocardial Infarction, Katus HA, Lindahl B, Morrow DA, Clemmensen PM, Johanson P, Hod H, Underwood R, Bax JJ, Bonow RO, Pinto F, Gibbons RJ, Fox KA, Atar D, Newby LK, Galvani M, Hamm CW, Uretsky BF, Steg PG, Wijns W, Bassand JP, Menasché P, Ravkilde J, Ohman EM, Antman EM, Wallentin LC, Armstrong PW, Simoons ML, Januzzi JL, Nieminen MS, Gheorghiade M, Filippatos G, Luepker RV, Fortmann SP, Rosamond WD, Levy D, Wood D, Smith SC, Hu D, Lopez-Sendon JL, Robertson RM, Weaver D, 
Tendera M, Bove AA, Parkhomenko AN, Vasilieva EJ, Mendis S. Third universal definition of myocardial infarction. Circulation. 2012;126(16):2020-2035.

15. National Health Service Summary Care Records. https://digital.nhs.uk/services/summary-care-records-scr. Accessed $7^{\text {th }}$ June 2018

16. Kahapola-Arachchige KM, Hadlow N, Wardrop R, Lim EM, Walsh JP. Age-specific TSH reference ranges have minimal impact on the diagnosis of thyroid dysfunction. Clin Endocrinol. 2012;77(5):773-779.

17. Hollowell JG, Staehling NW, Flanders WD, Hannon WH, Gunter EW, Spencer CA, Braverman LE. Serum TSH, T(4), and thyroid antibodies in the United States population (1988 to 1994): National Health and Nutrition Examination Survey (NHANES III). J Clin Endocrinol Metab. 2002;87(2):489-499.

18. Nicoloff JT. A new method for the measurement of thyroidal iodine release in man. J Clin Invest. 1970;49(10):1912-1921.

19. Weeke J, Gundersen HJ. Circadian and 30 minutes variations in serum TSH and thyroid hormones in normal subjects. Acta Endocrinol. 1978;89(4):659-672.

20. Sviridonova MA, Fadeyev VV, Sych YP, Melnichenko GA. Clinical significance of TSH circadian variability in patients with hypothyroidism. Endocr Res. 2013;38(1):24-31.

21. Persani L, Terzolo M, Asteria C, Orlandi F, Angeli A, Beck-Peccoz P. Circadian variations in thyrotropin bioactivity in normal subjects and patients with primary hypothyroidism. J Clin Endocrinol Metab. 1995;80(9):2722-2728.

22. Andersen IB, Brasen CL, Christensen H, Noehr-Jensen L, Nielsen DE, Brandslund I, Madsen JS. Standardised resting time prior to blood sampling and diurnal variation associated with risk of patient misclassification: results from selected biochemical components. PLoS One. 2015;10(10):e0140475. doi: 10.1371/journal.pone.0140475 
23. Wang D, Li D, Guo X, Yu S, Qiu L, Cheng X, Xu T, Li H, Liu H. Effects of sex, age, sampling time, and season on thyroid-stimulating hormone concentrations: a retrospective study. Biochem Biophys Res Commun. 2018;506(3):450-454.

24. Soeiro AM, Araujo VA, Vella JP, Bossa AS, Biselli B, Leal TCAT, Soeiro MCFA, Serrano CV Jr, Mueller C, Oliveira Junior MT. Is there any relationship between TSH levels and prognosis in acute coronary syndrome? Arq Bras Cardiol. 2018;110(2):113-118.

25. Rhee CM, Bhan I, Alexander EK, Brunelli SM. Association between iodinated contrast media exposure and incident hyperthyroidism and hypothyroidism. Arch Intern Med. 2012;172(2):153-159. 


\section{Figure legends}

Figure 1. The relationship between time of sampling over 24 hours and thyroid function parameters and TPOAb levels.

Restricted cubic spline plots with three knots demonstrating the association of time of sampling ( $x$ axis) with the predicted mean thyroid function and TPOAb parameters (black lines) with 95\% confidence intervals (grey area). All associations adjusted for age, sex, centre, body mass index, smoking status, type of AMI, pain to sampling time, serum creatinine, history of IHD, DM, CVD, BP, AF and hypothyroidism.

Figure 2. Risk of being diagnosed with subclinical hypothyroidism (A) or subclinical hyperthyroidism (B) in relation to time of sampling. Restricted cubic spline curves with three knots of log relative odds risk of being diagnosed with subclinical hypothyroidism (SCH) (A) or subclinical hyperthyroidism (SHyper) (B) (black line) with 95\% confidence intervals (grey areas) were plotted against time of sampling (24 hour clock), adjusted for age, sex, centre, body mass index, smoking status, type of AMI (STEMI or NSTEMI), pain to sampling time, serum creatinine, TPOAb levels, history of ischaemic heart disease, diabetes mellitus, hypertension, cerebrovascular disease and atrial fibrillation.

Figure 3. Time of sampling and the risk of remaining in subclinical hypothyroidism state.

Figure 4. Hazard plots of all-cause mortality in relation to baseline thyroid status determined using uniform TSH reference intervals (A) or time-period specific TSH reference intervals (B). $\mathrm{SCH}=$ subclinical hypothyroidism, SHyper = subclinical hyperthyroidism .

Table 1. Baseline characteristics and prevalence of thyroid dysfunction by time of blood sampling 
Figure 1. The relationship between time of sampling over 24 hours and thyroid function parameters and TPOAb levels.

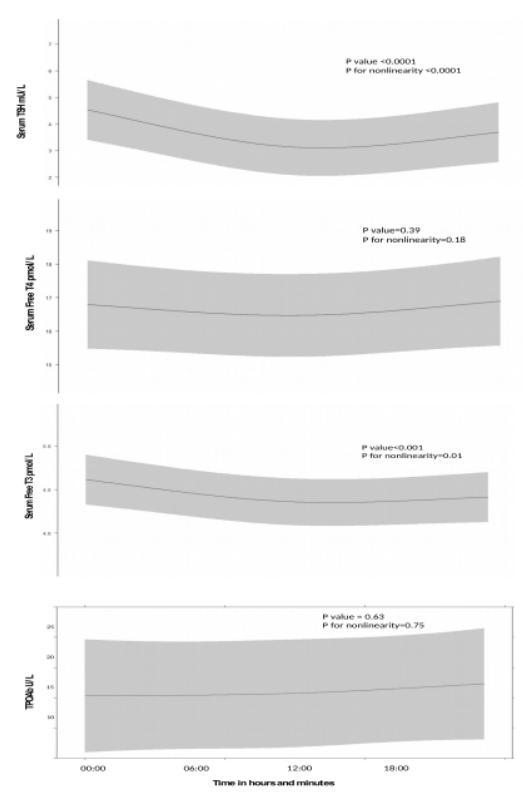


A

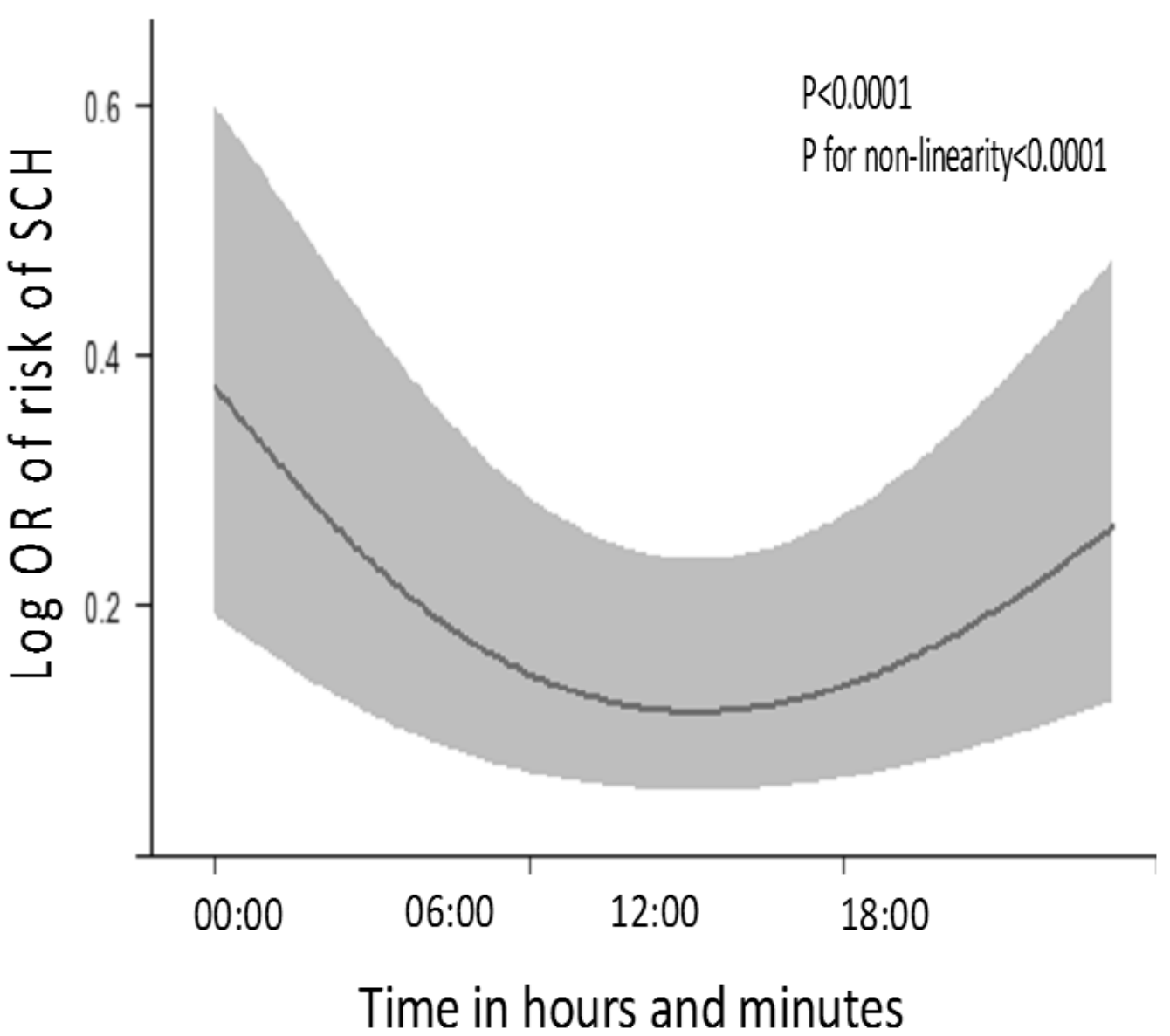

B

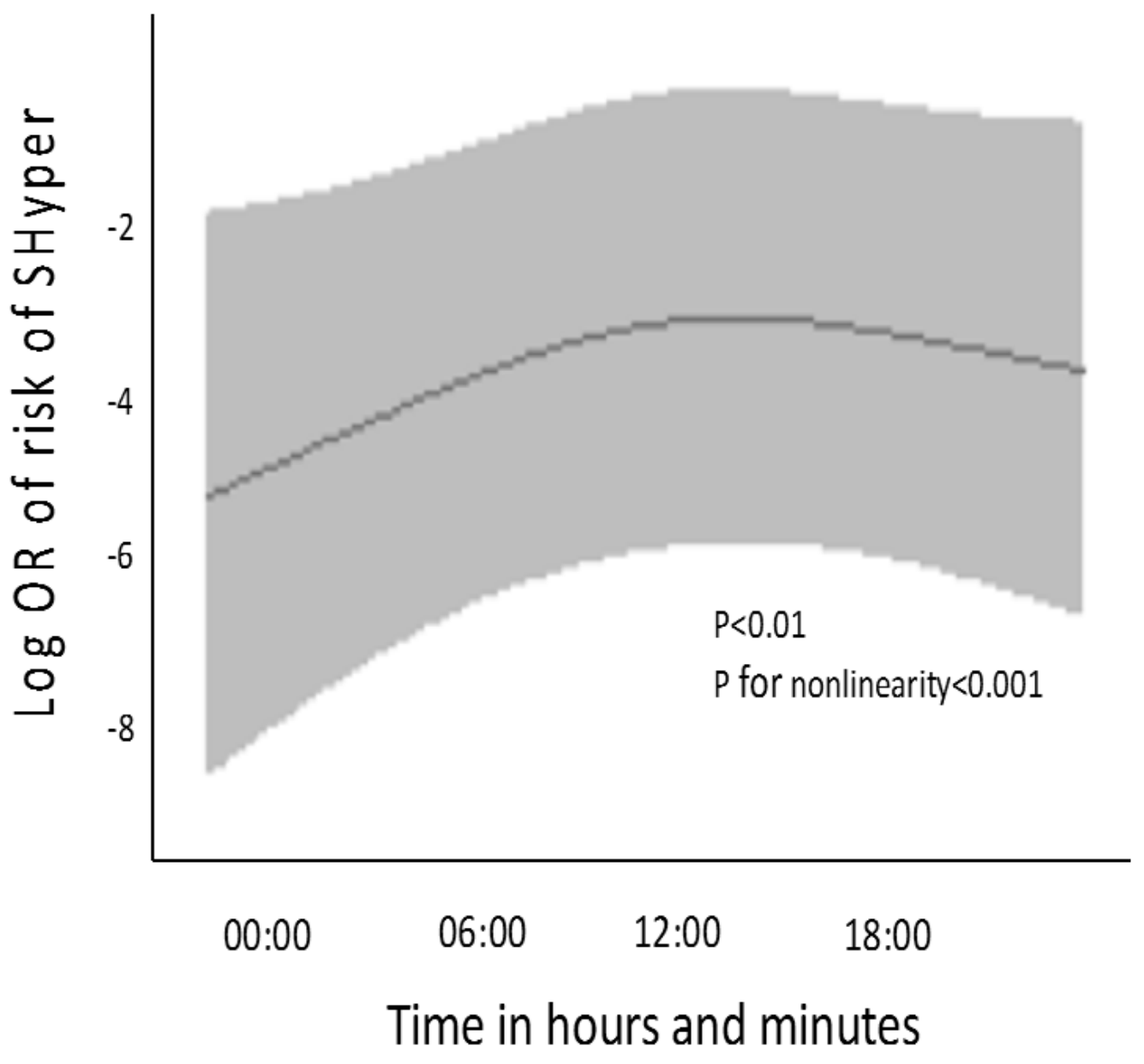


Figure 3. Time of sampling and the risk of remaining in subclinical hypothyroidism state in participants not on levothyroxine.

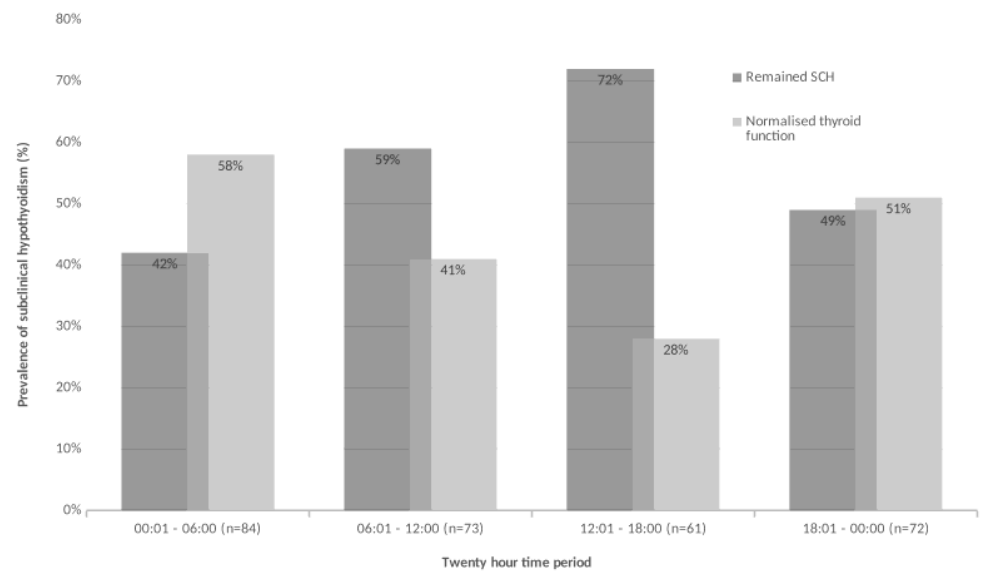


A

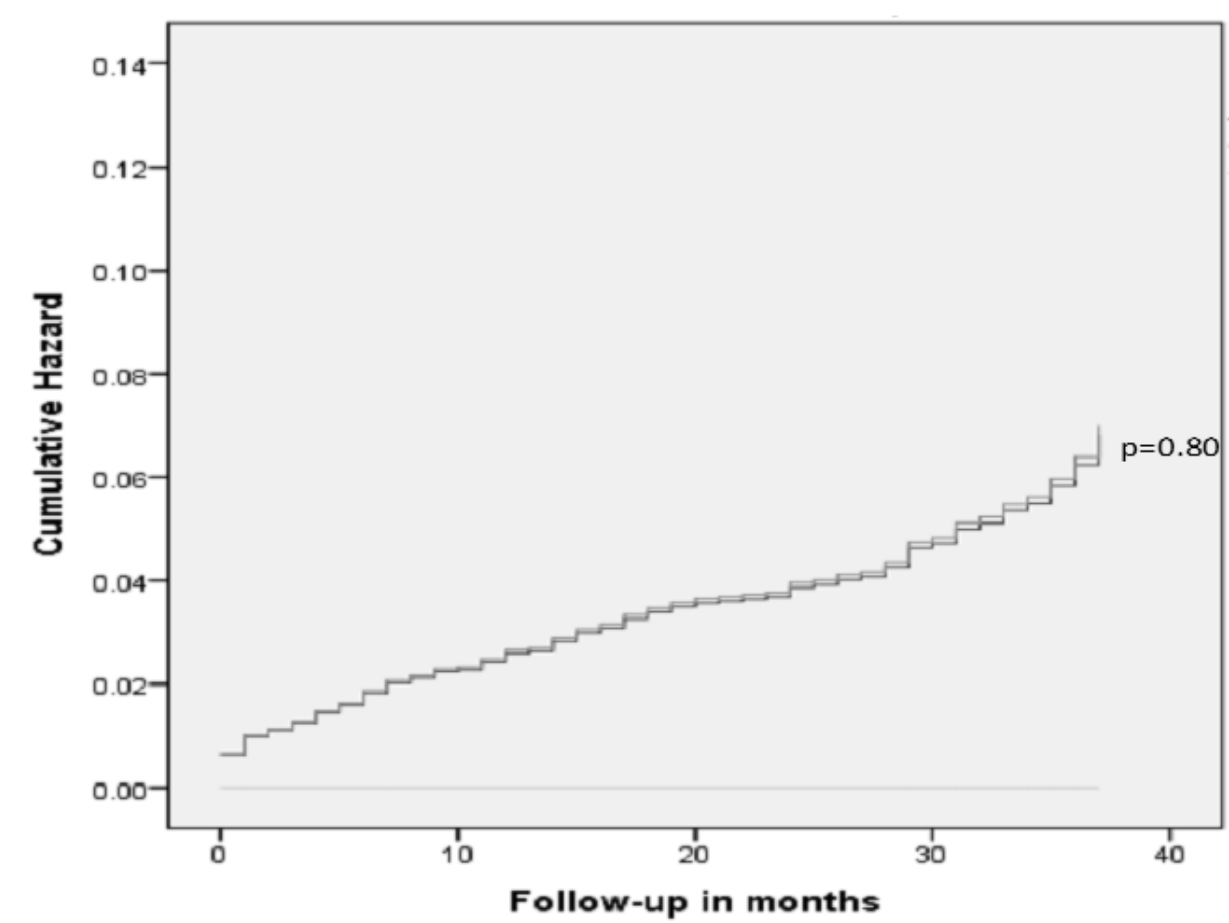

No. at risk

Euthyroid

SCH

SHyper

1470
313
23

1403
297
23

\begin{abstract}
1288
273
22
\end{abstract}

634
133

133
6
B

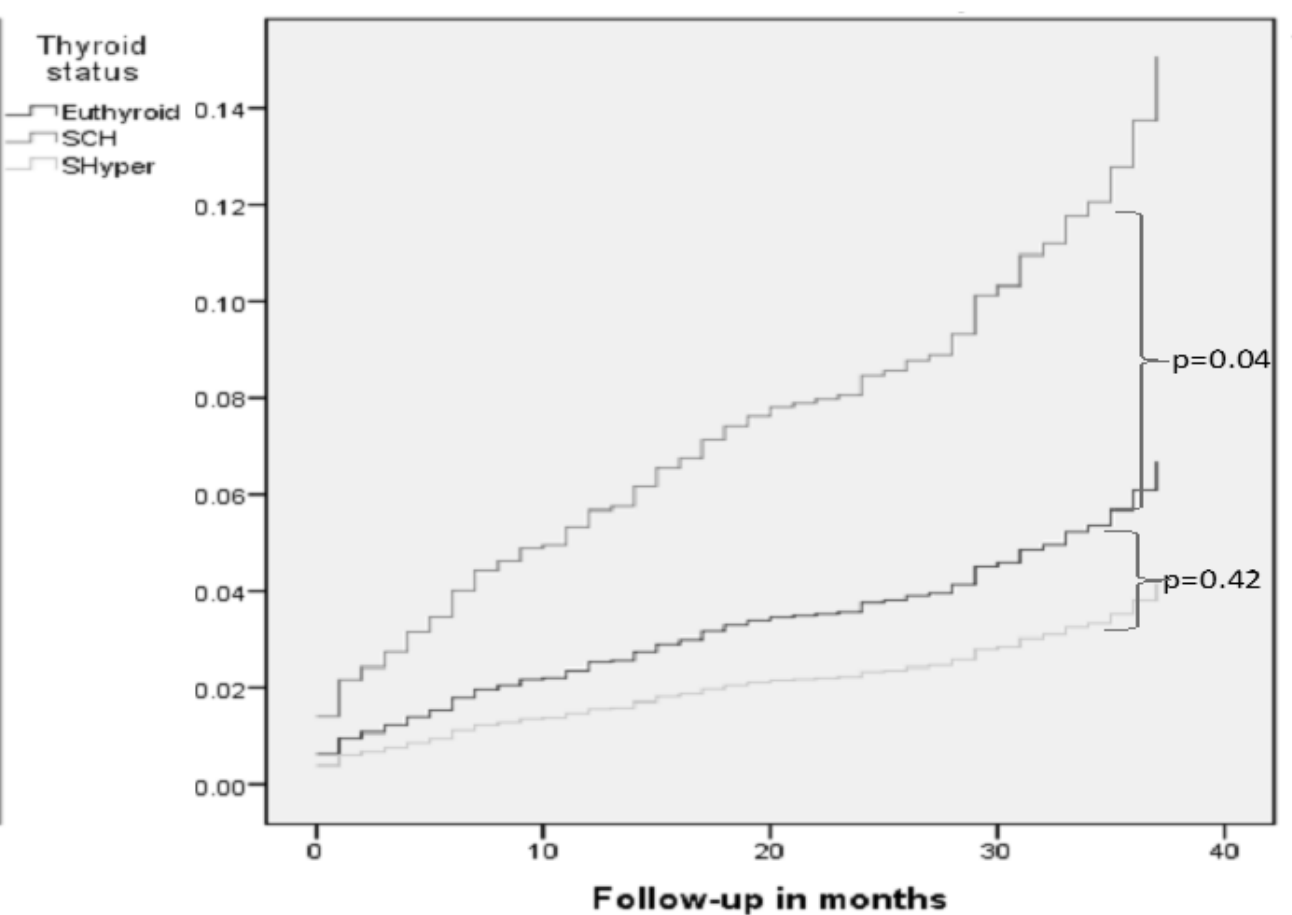

Follow-up in months

$\begin{array}{rrrrr}1720 & 1650 & 1512 & 743 & 59 \\ 49 & 38 & 37 & 17 & \\ 37 & 34 & 33 & 12 & 3\end{array}$

$\mathrm{SCH}=$ subclinical hypothyroidism, SHyper = subclinical hyperthyroidism . 